\title{
Research on the influence of team i-deals level on team innovation-from the perspective of collective thriving
}

\author{
Weiwei $\mathrm{Hu}^{1}$, Di Zheng ${ }^{1, *}$ \\ ${ }^{1}$ Zhejiang Gongshang University School of Business Administration Hangzhou, Zhejiang, China
}

\begin{abstract}
Based on the social cognition theory, this study explores how the median value of i-deals group influences team innovation through collective thriving, and the moderating role of chaxu climate in it. Through the empirical analysis of 367 questionnaires (70 supervisors' questionnaires and 297 employees' questionnaires) from 70 teams, it is proved that collective thriving has an mediating effect between team i-deals and team innovation, while chaxu climate positively regulates the positive impact of collective thriving on team innovation, that is, the higher the chaxu climate, the stronger the positive impact of collective thriving on team innovation.
\end{abstract}

\section{Introduction}

With the continuous upgrading of globalization and the flat development of organizational structure, teams have gradually become the basic work units for enterprises to cope with the challenges of competition ${ }^{[1]}$. Especially in the innovation activities, many successful enterprises regard teams as the main force of innovation because of the integration ability that individuals cannot achieve and the flexibility that enterprises do not have. A great deal of research has been done on how to stimulate team innovation, and the level of team resources is considered as an important driving force. Among them, the i-deals owned by the individual of the team is considered as an important resource of the team, because the personalized work arrangement not only meets the external requirements of the employees, but also meets the internal needs of the employees ${ }^{[2]}$.

Previous studies mainly focused on the analysis at the individual level, but the potential impacts of idiosyncratic deals(i-deals) at the group and organizational levels were relatively rare. Anand ${ }^{[3]}$ pointed out that the high-level team contract indicates that most members of the team can get customized learning opportunities, which enhances the sense of authorization and the ability and confidence of members to complete tasks. It can be seen that i-deals not only affects the results at the individual level, but also has a collective impact. In addition to the resources owned by the team, team atmosphere is an important environmental factor for team innovation. As a shared cognition of team members, it will form a team psychological state and play a role in shaping employee behavior ${ }^{[4]}$. However, this paper mainly studies the "localized" team situation factor-chaxu climate. In China, social circle culture is widespread, and team members will form a dense relationship around team leaders, which is manifested by the difference between "quality" and "quantity" in the relationship between different team members and team leaders, that is, the difference order. The characteristics of circle culture, superior and inferior power, differential treatment, private resource allocation, reciprocal social exchange and dynamic relationship highlighted by chaxu climate are a highly concrete summary of interpersonal relationships in Chinese society, and are "localized" situational factors worthy of attention and study ${ }^{[5]}$.

The main purpose of this paper is to explore whether the team level of i-deals is related to positive organizational results, and to study the theoretical mechanism behind these relationships. In this study, we pay attention to the relationship between i-deals and a key organizational result-team innovation. i-deals is considered to better meet the internal needs of employees, thus encouraging them to innovate. Therefore, they may also contribute to team innovation.

Based on the above discussion, this study has made contributions to the literature of i-deals group median and team innovation in three important aspects. Firstly, we investigated whether the median value of i-deals group is related to team innovation, so as to improve our understanding of the influence of the median value of i-deals group on team innovation. Secondly, we investigated whether the median value of $i$-deals group is related to collective thriving, and the mediating effect of collective thriving between the median value of $i$-deals group and team innovation, so as to understand the mechanism of the median value of $i$-deals group on team innovation. Finally, we integrate the literature of team innovation and chaxu climate to discuss the moderating effect of chaxu climate. We not only provide a theoretical framework of how the median value of i-deals group affects organizational results, but also study the moderating role of chaxu climate in this process.

*Corresponding author: 812432535@qq.com 


\section{Research design}

\subsection{Research hypothesis}

\subsubsection{Research hypothesis of median value of i-deals group on team innovation}

The median value of $i$-deals group represents the average level of i-deals within the team ${ }^{[3,6]}$. The median score of individual i-deals in an organization is used to describe the concentration trend among i-deals in an organization $^{[7-8]}$. According to Anand's research ${ }^{[3]}$, the high-level i-deals group median indicates that most members of the team can get customized learning opportunities, which enhances members' sense of authorization and their ability and confidence to complete tasks; the team with high median value indicates that members are treated equally, the possibility of harmony and cooperation among members is high, and group members are more inclined to share cognition ${ }^{[9]}$; the high group median also means a supportive environment, leaders care about employees' personal development, and leaders and employees respect and influence each other, so as to improve team effectiveness and team ability ${ }^{[10]}$. Anand's research ${ }^{[11]}$ found that the higher the overall contract level, the more beneficial it is to team cooperation and promote team performance, At this time, high-quality interpersonal relationships characterized by mutual respect and trust widely exist among members, and their psychological empowerment and self-efficacy are high ${ }^{[12]}$. The team has higher overall efficiency and lower team conflict, and members are more inclined to share cognition and have higher team ability when performing tasks ${ }^{[13]}$. Therefore, according to the above analysis, this paper thinks that i-deals group median can promote team innovation level.

H1: The median value of i-deals group has a significant positive impact on team innovation.

\subsubsection{Research hypothesis of median value of individualized contract group on collective thriving}

Spreitzer (2005) ${ }^{[14]}$ defined thriving as a psychological state in which individuals can perceive learning while perceiving vitality. Collective thriving refers to the continuous learning and growth of a team or organization as a whole ${ }^{[15]}$. According to the theory of social cognition, everyone has the possibility to experience the collective "vitality" and "learning", but the individual's thriving depends largely on the embedded working situation. Work situation is a powerful force to promote or consume collective thriving ${ }^{[16-17]}$. Anand ${ }^{[3]}$ believes that the median value of highly i-deals group represents a supportive working environment, which is full of mutual respect and influence, and leaders care about the personal development of employees. The social embeddedness model of Spreitzer ${ }^{[14]}$ shows that the atmosphere of trust and respect is an important precondition for job thriving. Individual job satisfaction brought by i-deals is likely to spread among employees, In organizations with high level of i-deals, higher collective job satisfaction will be produced ${ }^{[6]}$, while high level of collective job satisfaction means higher level of team vitality ${ }^{[18]}$. Median value of i-deals group promotes team effectiveness through team cooperation $^{[19]}$, and team cooperation ability is the most powerful predictor of thriving ${ }^{[16]}$. In teams with high i-dealss, employees get more valuable resources, and they tend to devote themselves to their work ${ }^{[20]}$, and devote themselves to making better performance, so team members are more likely to experience collective "vitality" and "learning".

H2: The median value of i-deals group has a significant positive impact on collective thriving.

\subsubsection{Research hypothesis of collective thriving on team innovation}

Innovation is an inexhaustible motive force for an organization to flourish,In order to gain sustainable competitive advantage in a rapidly changing environment, innovation frequently appears on the organizational agenda $^{[21]}$. Team innovation means that teams introduce and use new ideas, processes and products to benefit individuals, teams, organizations and society as much as possible. According to Van Knippenberg ${ }^{[22]}$, the existing research on the influencing factors of team innovation is mainly carried out from two perspectives: first, from the perspective of team atmosphere, innovation is regarded as the result of team atmosphere variables such as supporting innovation, sharing goals, task orientation and participation safety. Second, from the perspective of knowledge integration, the team is regarded as the natural home of information integration process, and the integration of different information, knowledge, ideas or viewpoints will bring new insights and stimulate team innovation, which is mainly reflected in the research on team information sources (emphasizing team diversity) and team information integration (emphasizing team interaction process). Collective thriving can be regarded as a special team atmosphere, and there is an atmosphere of continuous learning and growth in the prosperous collective ${ }^{[15]}$,Employees will have more energy to try to learn new methods, develop new technologies, design new workflow, etc, through different channels, and engage in more challenging work, so as to adapt to the dynamic work needs and achieve a prosperous state of work. Wu Jiangqiu et al., found through research that employees with prosperous work are more willing to spread the new knowledge and new ideas they have learned to the organization, and have more energy to put them into practice, thus significantly improving the innovation performance of employees ${ }^{[23]}$, which is conducive to the generation of team innovation.

H3: collective thriving has a significant positive impact on team innovation.

\subsubsection{The mediator role of collective thriving}

Prosperous teams or organizations are more resilient and flexible, facing uncertain environment, and can quickly respond to external changes ${ }^{[24]}$. Spreitzer(2007) ${ }^{[15]}$ found 
that the prosperous collective is not afraid to try new things, dare to take risks and learn from mistakes, which enhances the ability to solve new problems through improvisation or reorganization. Prosperous collectives have a high level of vitality by increasing activities, perseverance and innovation, which helps them to cope with obstacles, challenges, setbacks and failures, and stick to their efforts, that is, the energy contained in prosperous collectives helps to enhance the ability to cope with challenges, problems and crises, and is more resilient in the face of adversity or difficulties ${ }^{[24]}$. In addition, when the members of the unit are prosperous, they are more likely to help each other complete tasks, "thriving" helps people understand what they are doing and how to do it, and enhance their working ability and adaptability ${ }^{[25]}$. According to the above analysis, combined with assumptions 2 and 3 , it is reasonable to think that collective thriving is the explanation mechanism of the positive influence of the median value of i-deals group on team innovation.

H4: collective thriving plays an intermediary role between the median value of i-deals group and team innovation.

\subsubsection{The moderator role of chaxu climate}

In Chinese society, "circle phenomenon" and the pattern of difference order are ubiquitous and play an important role in team operation. The differential atmosphere refers to the difference degree of the relationship between team members around the master of team resources (usually team leaders $)^{[26]}$. The strong atmosphere of difference order means that different team members have great differences in the quantity and quality of resources obtained from the leaders. A few people can approach the center of power and resources, and employees within the organization are divided into different circles, which are divided into "insiders" and "outsiders". The weak atmosphere of difference order means that the resources obtained by different subordinate members are less different in nature and quantity, and the resources mastered by team members are the same, and employees and employees, employees and leaders are in a relaxed, equal and fair environment ${ }^{[26]}$. Studies have shown that chaxu climate does not necessarily make employees feel unfair, but has an incentive effect on employees.

When an employee perceives a high differential atmosphere, if he thinks that he is an "insider", he will do more civic behaviors to maintain his identity as an "insider", and if he feels that he is an "outsider", he will do more civic behaviors to manage his image in order to enter the circle or gain more recognition. At this time, they will realize that all resources in the organization are allocated according to the density of the relationship with the leaders, and they will put themselves in a favorable position in order to obtain resources to promote their organizational work. The research of Liu Jiaxin et al., ${ }^{[27]}$ shows that in the face of differential leadership, in order to gain the appreciation and resources of the leadership, subordinates will actively express their abilities, cooperate with the needs and goals of the organization, and establish a good interactive relationship with the leadership. The leader's partial treatment of his own subordinates affirms the contribution of his own subordinates and can encourage them to continue to take behaviors conducive to leadership effectiveness and team effectiveness. At the same time, it also sets an example for outsiders and encourages them to work hard to become their own subordinates ${ }^{[28]}$. Therefore, in the situation of high chaxu climate, the thriving level of the whole team is higher, and then the team innovation level will be higher.

H5: The chaxu climate regulates the positive influence of collective thriving and team innovation, the higher the chaxu climate, the stronger the positive influence of collective thriving on team innovation.

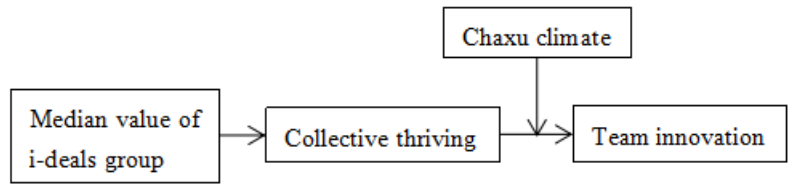

Figure 1 Research model

\subsection{Methods}

\subsubsection{Samples}

In this study, 83 supervisor questionnaires and 415 paired sample data of employees were collected. In the process of data entry, the unqualified questionnaires were deleted, and the final valid samples were 70 supervisors' questionnaires and 297 employees' questionnaires, which were in line with the median research object of i-deals group (team contract level). Among the investigated samples, men accounted for $56.9 \%$ and women accounted for $43.1 \%$, there are 153 undergraduates and 19 masters, accounting for $51.5 \%$ and $6.4 \%$. Most of the respondents came from Internet and financial industry, accounting for $44.4 \%$ of the total sample size, and there were 138 technical staff, accounting for $46.5 \%$ of the total sample size.

\subsubsection{Measures}

The following scales are measured by Likert's 5-point scoring method, where 1 means very different and 5 means very agree.

Personalize the values in the contract group. According to previous scholars' research, i-deals includes four dimensions: task, development, flexibility and salary. Rosen et al., are used to measure task i-deals, flexible i-deals and salary i-deals. The scale developed by (2013) includes 16 items, and the scale developed by Hornung, Rousseau, \& Glaser includes 4 items. The median score of $\mathrm{i}$-deals group is expressed by calculating the median score of individual level i-deals of each team, because the median score of i-deals can better reflect the concentration trend of team i-deals ${ }^{[3,6]}$. The reliability coefficient is 0.905 .

collective thriving. collective thriving adopts Porath et al., (2012) developed 10 items to measure the thriving 
of employees' workplaces, including 5 study items and 5 vitality items. The reliability coefficient is 0.912 .

Team innovation. Employees' innovative behavior uses six items developed by Scott \& Bruce(1994) to measure employees' innovative behavior in the workplace. The reliability coefficient is 0.901 .

chaxu climate. 11 items developed by Liu Zhenyu (2003) were used in the chaxu climate, this scale was first published in academic journals after Liu Jun and others passed the exploratory factor analysis test in 2009 . The reliability coefficient is 0.901 .

Control variable. Previous studies have shown that gender diversity, age diversity, educational diversity and team size will affect team process and team innovation. Therefore, the above four variables are treated as control variables in this study.

\section{Result}

\subsection{Data convergence analysis}

We use the average method to aggregate. Before convergence, HLM 6.08 software should be used to analyze the convergence validity, and the average rwg of collective thriving is $0.75, \operatorname{ICC}(1)$ is $0.12, \operatorname{ICC}(2)$ is 0.35 ; the mean rwg of team innovation is 0.75 , ICC( 1$)$ is 0.12 , $\operatorname{ICC}(2)$ is 0.35 ; rwg mean of chaxu climate is 0.76 , ICC(1) is 0.13 , $\operatorname{ICC}(2)$ is 0.36 . Rwg mean is greater than 0.7 , $\operatorname{ICC}(1)$ is greater than 0.1 , which indicates that each variable has enough variance at individual level and team level to explain, and that each variable can converge to team level and become a team level variable.

\subsection{Correlation analysis}

The median value of i-deals group is positively correlated with collective thriving $(\mathrm{r}=0.571, \mathrm{p}<0.01)$ and team innovation $(\mathrm{r}=0.450, \mathrm{p}<0.01)$. There is a significant positive correlation between collective thriving and team innovation $(\mathrm{r}=0.580, \mathrm{p}<0.01)$. However, there is a significant negative correlation between chaxu climate and collective thriving $(\mathrm{r}=-0.700, \mathrm{p}<0.01)$ and team innovation $(\mathrm{r}=-0.512, \mathrm{p}<0.01)$. In addition, there is no significant correlation between gender diversity, age diversity, education diversity and team size and the median value of i-deals group, which indicates that the median value of i-deals group has little difference among teams of different ages, genders, educational levels and sizes.

\subsection{Regression analysis}

In Model 4, the median value of i-deals group has a significant positive impact on team innovation $(\beta=0.419$, $\mathrm{p}<0.01$ ), and Hypothesis 1 holds. In Model 2, the median value of i-deals group has a significant positive impact on collective thriving $(\beta=0.578, \mathrm{p}<0.001)$, and Hypothesis 2 holds. In model 5 , collective thriving has a significant positive impact on team innovation $(\beta=0.545$, $\mathrm{p}<0.001$ ), and hypothesis 3 holds. In model 8 , the regression coefficient of the interaction between collective thriving and chaxu climate on team innovation is significant $(\beta=0.236, p<0.05)$, which indicates that chaxu climate positively regulates the positive correlation between collective thriving and team innovation. Therefore, hypothesis 5 holds. Value of i-deals group decreased from significant $(\beta=0.419$, $\mathrm{p}<0.01)$ to insignificant ( $\beta=0.152, \mathrm{p}>0.05)$, and collective thriving had a significant positive impact on team innovation ( $\beta=0.462, p<0.001$ ), indicating that in model 8 , the regression coefficient of the interaction between collective prosperity and differential order atmosphere on team innovation is significant $(\beta=0.236$, $\mathrm{p}<0.05$ ), which indicates that differential order atmosphere positively regulates the positive correlation between collective prosperity and team innovation. Therefore, hypothesis 5 holds.

In order to further test the significance of mediation effect, we used Bootstrap method (preacher\& Hayes, 2004) to further analyze the significance of mediation effect. The results show that the 95\% confidence intervals of the mediating effect of collective thriving are (0.0957, 0.4878), excluding zero. Therefore, the mediating effect of collective thriving is significant, and hypothesis 4 is further supported by the data.

In order to verify the moderating effect of chaxu climate in the research results, according to the suggestion of Aiken and West, the relationship between collective thriving and team innovation is drawn on the high and low levels of chaxu climate with the observed values of standard deviation +1 and -1 . Compared with low-level chaxu climate, high-level chaxu climate enhances the positive effect of collective thriving on team innovation. Therefore, hypothesis 5 is further supported by the data.

\section{Conclusion}

This paper takes the median value of i-deals group as antecedent variable, and explains the mechanism of the median value of i-deals group on team innovation by introducing collective thriving. As interpersonal relationship is an important environmental factor that affects team innovation, this paper introduces the chaxu climate as a moderating variable, and constructs and verifies a mediating moderating model. The conclusions are as follows: 1) The median value of i-deals group has a significant positive impact on team innovation; 2) Individualized contract group median has a significant positive impact on collective thriving, and collective thriving has a significant positive impact on team innovation, collective thriving completely mediates the positive impact of individualized contract group median on team innovation; 3) The effect of collective thriving on team innovation is influenced by chaxu climate, which positively regulates the relationship between them, the higher chaxu climate, the stronger the effect between them. 


\section{References}

1. Guo WX, Wang DX. Soft Science, 1, 68-71 (2014).

2. Rosen C C, Slater D J, Chang C H, et al., JOM , 2013, 39(3): 709-742.

3. Anand S. University of Illinois at Chicago, 2012.

4. Anderson N R, West M A. JOB , 19, 235-258(1998) .

5. Chen Zhixia, Dian Yajiao. Foreign Economy and Management, 6(2018).

6. Schuler A F, Bruch H. Academy of Management Annual Meeting Proceedings, 2018 (online).

7. Bliese P D . (2000) .

8. Henderson D J, Liden R C, Glibkowski B C, et al., The leadership quarterly, 20, 517-534(2009) .

9. Madjar N. Journal of Occupational and Organizational Psychology, 81, 83-100(2008) .

10. Boies K, Howell J M. The Leadership Quarterly, 17, 246-257 (2006).

11. Anand S, Meuser J D, Vidyarthi P R, et al., Academy of Management Proceedings. Briarcliff Manor, 1, (2017) .

12. Peng JP. South China University of Technology (Social Science Edition), 6, 12-17 (2012) .

13. Rousseau D M, Hornung S, Kim T G. JVB, 74 338-348 (2009).

14. Spreitzer G M, Sutcliffe K M, Dutton J, et al., Organization science, 16, 537-549 (2005) .

15. Spreitzer G M, Sutcliffe K M. Positive organizational behavior, 74-85 (2007) .

16. Spreitzer G M, Porath C L, Gibson C B. Organizational Dynamics, 41, 155-162 (2012) .

17. Spreitzer G M, Porath C. The Oxford handbook of work engagement, motivation, and self-determination theory, 245-258 (2014).

18. Zhong W, Zhang L. JOM, 379 (2010).

19. Liao C. Academy of Management Proceedings. Briarcliff Manor, NY 10510 , 1 (2014).

20. Zhang X, Fan Y, Huang MP, et al., Nankai business review, 59-69 (2013) .

21. West M A, Anderson N R. JAP, 81 (1996).

22. Van Knippenberg D, De Dreu C K W, Homan A C. JAP, 89 (2004) .

23. Wu JQ, Huang PL, Yan D. Soft Science, 110-113 (2015) .

24. Han Y, Wei WW. Foreign Economy and Management, 8 46-53 (2013) .

25. Walumbwa F O, Muchiri M K, Misati E, et al., JOB, 39, 249-261 (2018).

26. Liu J, Zhang K, Zhong LF. Managing the World, 92-101 (2009).

27. Liu JX, Qi M, Xu G. Leadership Science, 2616 (2017).

28. Chloe W. Advances in psychological science, 1688-1700 (2015) 